Proc. Indian Acad. Sci. (Chem. Sci.), Vol. 96, No. 6, April 1986, pp. 533-547. (C) Printed in India.

\title{
Ionic conduction in dispersed solid-electrolytes $\dagger$
}

\author{
A. K SHUKLA*, N VAIDEHI and K T JACOB $\S$ \\ Solid State and Structural Chemistry Unit, Indian Institute of Science, Bangalore 560012, \\ India \\ $\S$ Department of Metallurgy, Indian Institute of Science, Bangalore 560012, India
}

\begin{abstract}
In recent years, considerable interest has been focussed on developing solid electrolytes with high ionic conductivities. One of the latest techniques is the dispersion of a non-conducting and chemically non-interacting phase in the solid electrolyte. These eloctrolytes have been referred to as dispersed solid-electrolyte systems. The present paper briefly reviews the characteristic features of dispersed solid-electrolytes and the mode of ion conduction in these systems.
\end{abstract}

Keywords. Ionic conduction; solid-electrolytes; dispersed solid-electrolyte systems.

\section{Introduction}

One of the reasons for developing solid electrolytes is the need to minimize irreversible losses arising from the high internal resistances in traditional electrochemical devices with aqueous electrolytes. The reduction of internal resistances will assist in the attainment of high reaction rates and good transport conditions. One obvious way to reduce these resistances would be to operate the electrochemical devices at high temperatures. This, however, necessitates the use of ceramic materials having suitably high specific conductivities for ions which can take part in electrochemical processes. The development of such materials, called fast ion-conductors, has contributed to a breakthrough in electrochemical technology. In the literature (Rickert 1982), substantial amount of research and development work has been reported to achieve industrially viable fast ion-conductors. Starting from the discovery of high ionic conductivity in $\alpha$-AgI (Strock 1934, 1935) during the 1930 s, $\mathrm{Ag}_{3}$ SI (Reuter and Hardel 1961,1965 ), $\operatorname{RbAg}_{4} \mathrm{I}_{5}$ (Bradley and Greene 1966, 1967; Owens and Argue 1967) and $\beta$ alumina (Weber and Kummer 1967) in the 60s, Nasicons (Goodenough et al 1976) and Lisicons (Hong 1978) in the 1970s, the present day endeavours are in developing dispersed solid-electrolyte systems, wherein an ionically conducting matrix is dispersed with a chemically non-interacting and electrically non-conducting phase. Such solid electrolyte systems have been reported to exhibit significantly higher ionic conductivities in relation to pure host solid electrolytes. The present paper highlights some important facets of these solid-electrolytes.

\section{Historical background}

The development of dispersed solid-electrolytes could be linked to the observation made during the beginning of this century (Jander 1929) that the conductivity of two-

† Communication No. 352 from the Solid State and Structural Chemistry Unit.

* To whom all correspondence should be addressed. 


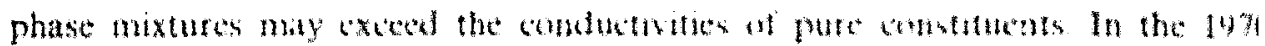

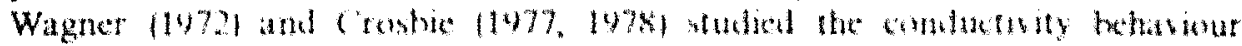

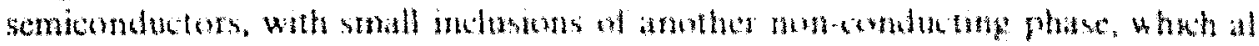

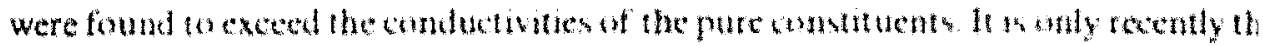

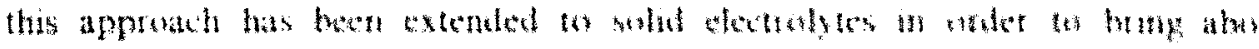
conancoments in their intic comluctivities

\section{Characteristic features}

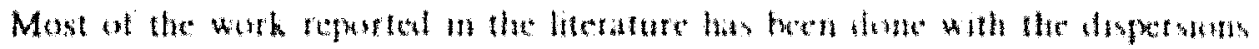

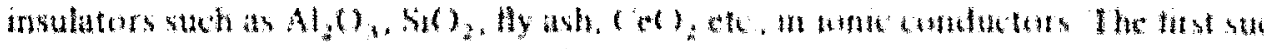

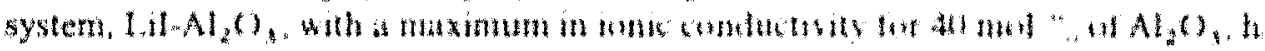

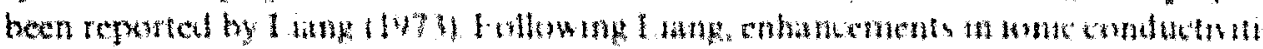

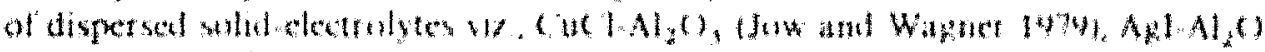

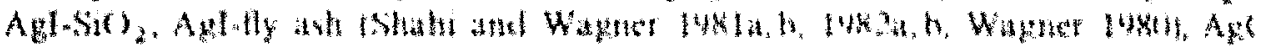

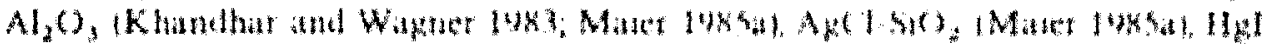

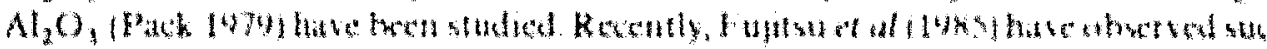

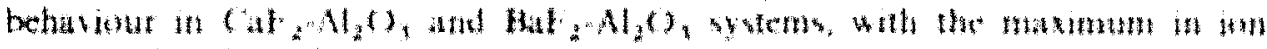

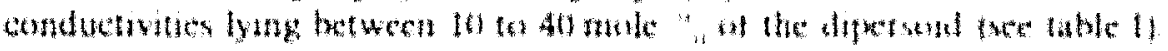

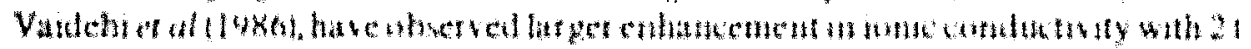

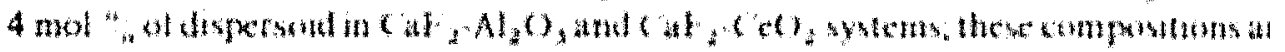

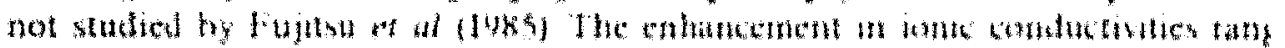

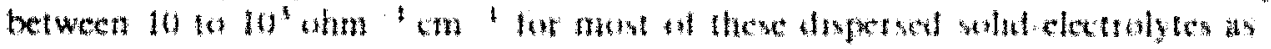

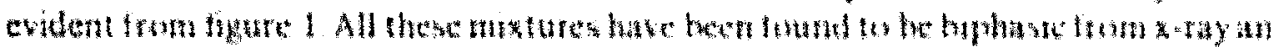

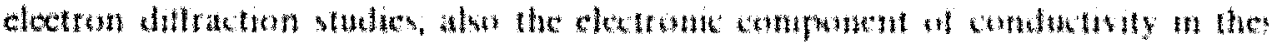

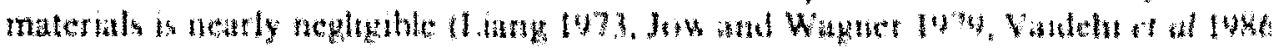

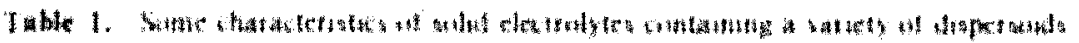

\begin{tabular}{|c|c|c|c|c|}
\hline Materal & 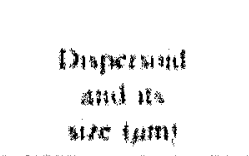 & 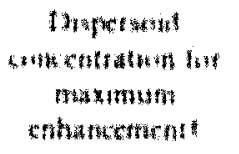 & 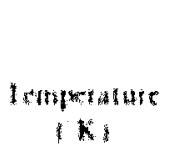 & 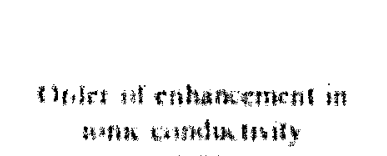 \\
\hline An & IJard $A, O, 11,4$ & Muta & then & 4 \\
\hline Agl & Myorated $\mathrm{Al}_{2} \mathrm{O}$ & Winn & $* u$ & $w^{*}$ \\
\hline Al & $\mathrm{SHC}, \mathrm{ethln}$ & Him a & $n^{4}+\sqrt{h}$ & 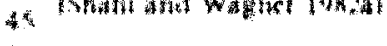 \\
\hline A & Nyant & 1154 & and & 4 \\
\hline L.11 & $A(1)$ & 4 alla & $7 x^{2}$ & 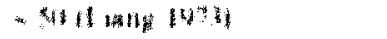 \\
\hline cul & $A_{2}()_{1}(t)(k)$ & $\operatorname{lom} a$ & I & * Hork Hod Wagnet Isol \\
\hline $114 t_{n}$ & $\left\|_{2}()_{3}\right\|(1)$ & Han & 34 & 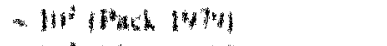 \\
\hline$A g$ & $(,, 1), 10)$ & $1+4$ & 3 & 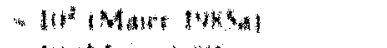 \\
\hline AqE & $446 y_{3}(12(x)$ & How & $2 / 4$ & 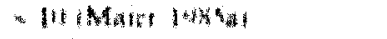 \\
\hline 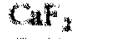 & 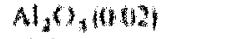 & $2 \pi / 0$ & $b 1$ & 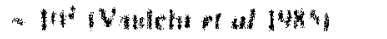 \\
\hline $\mathrm{Cat}^{2}$ & $6 \mathrm{CO}^{\circ} 10111$ & $2 n+1$ 年 & ofit & 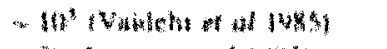 \\
\hline $\mathrm{Bat}_{2}$ & $\mathrm{Al}_{2} \mathrm{O}, \mathrm{g}, \mathrm{n}$, & whan & 71 & 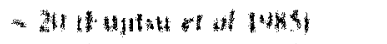 \\
\hline $\mathrm{CH}_{1}$ & $\left.A L_{3}()_{3}, 0\right)$ & UIm & 31 & 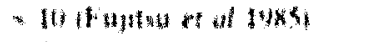 \\
\hline
\end{tabular}

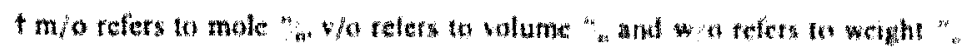




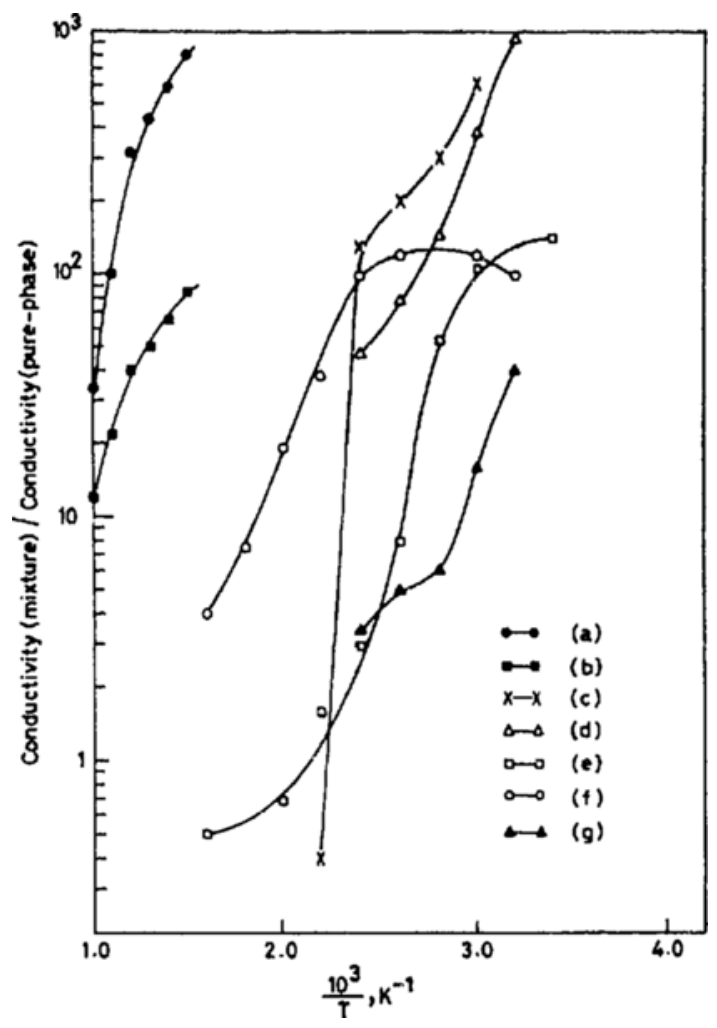

Figare 1. Enhancements in ionic conductivities for various mixtures as a function of temperature: (a) $\mathrm{CaF}_{2}-\mathrm{CeO}_{2}(2 \mathrm{~m} / \mathrm{o}, 0-01 \mu \mathrm{m})$, (b) $\mathrm{CaF}_{2}-\mathrm{Al}_{2} \mathrm{O}_{3}(2 \mathrm{~m} / 0,0-02 \mu \mathrm{m})$, (c) AgI$\mathrm{Al}_{2} \mathrm{O}_{3}(30 \mathrm{~m} / 0,0-06 \mu \mathrm{m})$, (d) $\mathrm{Agl}-\mathrm{AgBr}(20 \mathrm{~m} / \mathrm{o})$, (e) $\mathrm{AgCl}_{\mathrm{Al}} \mathrm{Al}_{3}$ ( $\left.3 \mathrm{v} / \mathrm{o}, 0-3 \mu \mathrm{m}\right)$, (f) CuCl$\mathrm{Al}_{2} \mathrm{O}_{3}(10 \mathrm{~m} / \mathrm{o}, 0.06 \mu \mathrm{m})$, and $(\mathrm{g}) \mathrm{AgI}$-flyash $(13.5 \mathrm{w} / \mathrm{o})(\mathrm{m} / \mathrm{o}=$ mole $\% ; \mathrm{v} / \mathrm{o}=$ volume $\%$; $\mathrm{w} / \mathrm{o}=$ weight $\%$ ).

The factors which produce such as enhancement in ionic conductivity of these dispersed solid electrolytes are:

(a) Temperature: As is evident from figure 1, the relative enhancements in ionic conductivity of the dispersed solid electrolytes, with respect to their pure phases, are substantially higher at low temperatures. Also, a decrease in activation energies for ion migration with respect to pure phases have been observed as shown in table 2 . The conductivities of the dispersed solid-electrolytes and those of the pure phases become almost the same at high temperatures in many systems like $\mathrm{AgCl}-\mathrm{SiO}_{2}$ (Maier 1985a), $\mathrm{LiI}-\mathrm{Al}_{2} \mathrm{O}_{3}$ (Liang 1973) and $\mathrm{CuCl}-\mathrm{Al}_{2} \mathrm{O}_{3}$ (Jow and Wagner 1979). However, for the systems $\mathrm{CaF}_{2}-\mathrm{Al}_{2} \mathrm{O}_{3}$ and $\mathrm{CaF}_{2}-\mathrm{CeO}_{2}$ (Vaidehi et al 1986) there is a break in the conductivity of the dispersed phase. For all temperatures where measurements have been made, the conductivity of the dispersed electrolyte in these systems is higher than that of pure $\mathrm{CaF}_{2}$. Difference between the conductivities of the dispersed and pure phases has also been observed upto the melting point for the AgI-AgBr system (Shahi and Wagner 1982b), in which there is solid solubility of the dispersed phase in the matrix. 
Table 2. Summary of electrical transport data.

\begin{tabular}{|c|c|c|c|}
\hline \multirow[b]{2}{*}{ Material/composition } & \multirow{2}{*}{$\begin{array}{c}\text { Defect } \\
\text { migration } \\
\text { energies }(e V)\end{array}$} & \multicolumn{2}{|c|}{ Defect formation energies (eV) } \\
\hline & & Vacancy & Interstitial \\
\hline AgI (pure) ${ }^{\mathrm{a}}$ & 0.56 & 0.3 & 0.14 \\
\hline $\mathrm{AgI}+30 \mathrm{~m} / 0$ hydrated $\mathrm{Al}_{2} \mathrm{O}_{3}{ }^{a}$ & $0 \cdot 26$ & & \\
\hline $\mathrm{AgI}+10 \mathrm{~m} / \mathrm{o} \mathrm{SiO}{ }_{2}^{2}$ & $0 \cdot 29$ & & \\
\hline $\mathrm{AgI}+13.5 \mathrm{w} / 0$ flyash ${ }^{\mathrm{a}}$ & $0 \cdot 26$ & & \\
\hline $\mathrm{AgCl}^{\mathrm{a}}$ & 0.8 & 0.29 & 0.045 \\
\hline $\mathrm{AgCl}+13 \mathrm{~m} / \mathrm{OAl}_{2} \mathrm{O}_{3} \mathrm{z}$ & $0 \cdot 31$ & & \\
\hline $\mathrm{AgCl}+11$ v/O $\mathrm{SiO}_{2}{ }^{\mathrm{a}}$ & $0 \cdot 30$ & & \\
\hline $\mathrm{AgBr}^{2}$ & 0.75 to 0.8 & 0.33 & 0.14 \\
\hline $\mathrm{AgBr}+10$ \% $/ 0 \mathrm{Al}_{2} \mathrm{O}_{3}{ }^{\mathrm{a}}$ & $0-35$ & & \\
\hline $\mathrm{CaF}_{2}{ }^{\mathrm{b}}$ & 0.91 & 0.51 & 0.92 \\
\hline $\mathrm{CaF}_{2}+2 \mathrm{~m} / \mathrm{OAl} \mathrm{Ol}_{3} \mathrm{~b}$ & $0-71$ & & \\
\hline $\mathrm{CaF}_{2}+2 \mathrm{~m} / \mathrm{o} \mathrm{CeO}_{2} \mathrm{~b}$ & 0.66 & & \\
\hline $\mathrm{LiI}^{\mathrm{a}}$ & 0.8 & & \\
\hline $\mathrm{LiI}+40 \mathrm{~m} / \mathrm{o} \mathrm{Al}_{2} \mathrm{O}_{3}{ }^{2}$ & 0.42 & & \\
\hline
\end{tabular}

after Maier 1985b; b after Vaidehi et al 1986.

(b) Concentration of the dispersoid: The ionic conductivities of dispersed solidelectrolytes generally exhibit a maximum at a certain concentration (see figure 2). A broad break, covering a large range of concentrations, has also been observed in $\mathrm{HgI}_{2}$ $\mathrm{Al}_{2} \mathrm{O}_{3}$ (Pack 1979; Pack et al 1980), and AgI-predried $\mathrm{Al}_{2} \mathrm{O}_{3}$ (Shahi and Wagner 1982a). It is noteworthy that unlike the case for solid electrolytes dispersed with hydrated alumina, the enhancements in the conductivities of solid electrolytes dispersed with predried alumina are insignificant. The role of hydroxyl ions in enhancing the ionic conductivities of the dispersed solid-electrolytes is hence not ruled out.

(c) Particle size of the dispersoid: The enhancement in ionic conductivities is a strong function of the particle size of the dispersoids. Figure 3 shows the variation of ionic conductivity with particle size for $\mathrm{CuCl}-10$ mole $\% \mathrm{Al}_{2} \mathrm{O}_{3}$ (Chang 1978) at $373 \mathrm{~K}$. The conductivity decreases with increase in particle size. Similar behaviour has also been reported (Shahi and Wagner 1982a) for other systems also. As is evident from figure 3, the conductivity enhancements for a given concentration of the dispersoid are large for small particles. Probably, the large dispersoid particles block the path for ion conduction in these solid electrolytes.

(d) Method of preparation: A dispersed solid-electrolyte system is prepared by mixing the two components -ionic conductor and insulator-followed by sintering. When the particle size of the dispersed solid is much smaller than that of the host matrix, the dispersed solid-electrolytes prepared by the powder method would contain most of the dispersoids along the grain boundaries. The sintering temperature is found to influence the conductivity of dispersed solid electrolyte. Figure 4 illustrates the variation of conductivity with calcining temperature for $\mathrm{CaF}_{2}-\mathrm{Al}_{2} \mathrm{O}_{3}$ (Fujitsu et al 1985) which exhibits a maximum at $1773 \mathrm{~K}$. Jow and Wagner (1979) have reported similar results for $\mathrm{CuCl}-\mathrm{Al}_{2} \mathrm{O}_{3}$ system. 


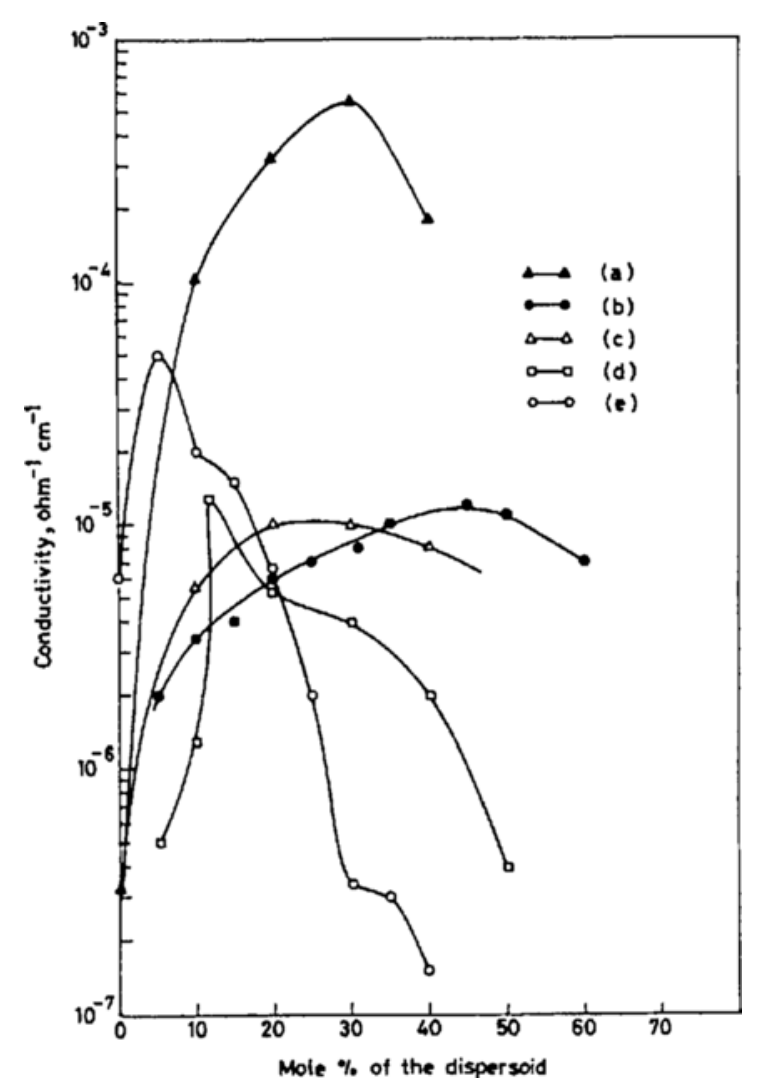

Figure 2. Variation of ionic conductivity with concentration of dispersiod for various dispersed solid-electrolytes: (a) AgI-hydrated $\mathrm{Al}_{2} \mathrm{O}_{3} \quad\left(25^{\circ} \mathrm{C}, 0.06 \mu \mathrm{m}\right)$, (b) $\mathrm{LiI}-\mathrm{Al}_{2} \mathrm{O}_{3}$ $\left(25 \pm 2^{\circ} \mathrm{C}\right), \quad$ (c) AgI-predried $\mathrm{Al}_{2} \mathrm{O}_{3} \quad\left(25^{\circ} \mathrm{C}, 0.06 \mu \mathrm{m}\right), \quad$ (d) Agl-flyash $\quad\left(24^{\circ} \mathrm{C}\right)$, and (e) $\mathrm{CaF}_{2}-\mathrm{Al}_{2} \mathrm{O}_{3}\left(500^{\circ} \mathrm{C}, 0.06 \mu \mathrm{m}\right)$.

\section{Mode of conduction}

In order to explain the conductivity behaviour of biphasic mixtures of materials several theoretical models have been advanced in the literature (Rayleigh 1892; Lichtenecker 1924; Landauer 1952; Maxwell 1965; Wagner 1972; Crosbie 1977, 1978; Pack 1979; Jow and Wagner 1979; Stoneham et al 1979; Maier 1984; 1985a, b). A number of different shapes and arrangements of the mixture can be visualized. Conductivities of a bimetallic mixture frequently expressed in metallurgical literature (Landauer 1952) are

$$
\rho_{M}=x_{1} \rho_{1}+x_{2} \rho_{2},
$$

where $\rho_{M}$ is the resistivity of the mixture; $x_{1}$ and $x_{2}$ are volume fraction of materials 1 and 2 respectively; $\rho_{1}$ and $\rho_{2}$ are their respective resistivities. This would be correct if the materials were arranged in alternate layers, perpendicular to the direction of current flow. In such a model, the current cannot avoid the regions of high resistances, as it will do if the regions are mixed at random. Also, the approximation is adequate only if the 


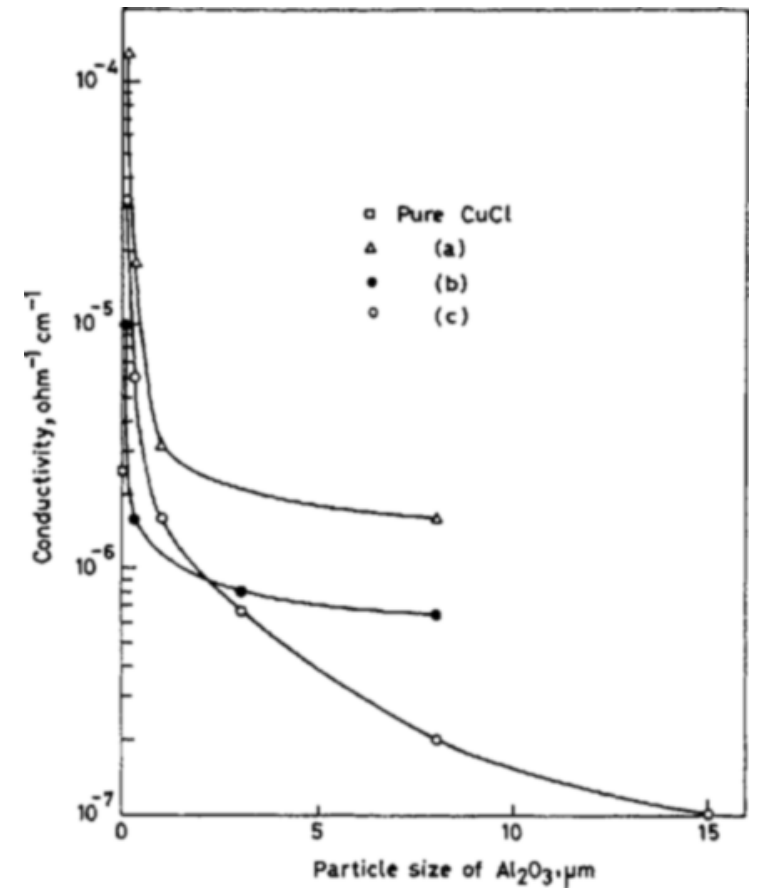

Figure 3. Variation of conductivity with the particle size of $\mathrm{Al}_{2} \mathrm{O}_{3}$ for various host matrices: (a) $\mathrm{HgI}_{2}-25 \mathrm{~m} / \mathrm{o}$ hydrated- $\mathrm{Al}_{2} \mathrm{O}_{3}\left(203^{\circ} \mathrm{C}\right)$, (b) $\mathrm{AgI}-30 \mathrm{~m} / \mathrm{o}$ predried $-\mathrm{Al}_{2} \mathrm{O}_{3}\left(25^{\circ} \mathrm{C}\right)$, and (c) $\mathrm{CuCl}-10 \mathrm{~m} / \mathrm{o} \mathrm{Al} \mathrm{O}_{3}\left(100^{\circ} \mathrm{C}\right)$.

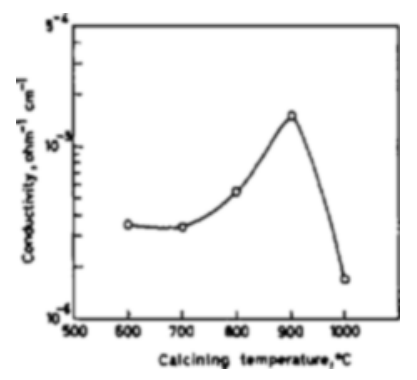

Figure 4. Effect of calcining temperature on conductivity for $\mathrm{CaF}_{2}-20 \mathrm{~m} / \mathrm{oAl} \mathrm{Al}_{2} \mathrm{O}_{3}$ of $0-3 \mu \mathrm{m}$ particle size.

two phases are passive. Therefore, (1) will give an upper limit for the resistivity. If the layers are parallel to the direction of current flow, then the total conductivity of the mixture, $\sigma_{M}$, will be given by

$$
\sigma_{M}=x_{1} \sigma_{1}+x_{2} \sigma_{2}
$$

Equation (2) will give an upper limit for the conductivity. The current can therefore flow straight through the regions of low resistivity preferentially, without having to follow a warped course, as will be the case in a random mixture. 
Another approximation, which agrees better with the measured values of the resistance of metallic mixtures was put forth by Lichtenecker (1924):

$$
\rho_{M}=\rho_{1}^{x_{1}} \rho_{2}^{x_{2}}
$$

Equation (3) may be considered as an improvement over (1) as it incorporates the nonpassive components of the two chemical phases to a certain extent. In comparing the results of existing theories with experimental results it becomes apparent that all methods of interpolating between $\sigma_{1}$ and $\sigma_{2}$ or between $\rho_{1}$ and $\rho_{2}$ give good results if the two conductivities are of the same order of magnitude. The validity of such theories can only be critically tested by considering the case where the conductivities $\sigma_{1}$ and $\sigma_{2}$ differ appreciably. It can easily be seen that (3) does not meet this requirement. If there is only a very small amount of material 2 with $\rho_{2}=0$, (3) gives $\rho_{M}=0$. Actually, a few small particles of perfect conductor would not alter the conductivity of a medium appreciably, since most of the current's path would still be in a medium of finite conductivity. Similarly a small trace of 2 with $\rho_{2}=\infty$ would give $\rho_{M}=\infty$ which is unreasonable.

Rayleigh (1892) treated the electrical conduction in biphasic systems by considering that material 2 exists in the form of spheres or cylinders, forming a rectangular array which is embedded in material 1 (figure 5). In this model, there exist lines of current flow which stay entirely within material 1 and are never interrupted by meeting an obstacle of material 2. On the other hand, current can never flow from a piece of material 2 to another piece of the same material without traversing a region of material 1 . Therefore, even if there are equal volumes of 1 and 2 , it will primarily be the conductivity of material 1 that predominates. Rayleigh's treatment is accurate only if the amount of material 2 is small compared to that of 1 or if the situation is one in which material 1 always envelops material 2. Physical arrangement in real systems rarely corresponds to the idealization in Rayleigh's model.

Wagner (1972) attempted to explain the conductivity behaviour of biphasic semiconductor-metal mixtures, such as $\mathrm{Zn}$-metal dispersed in $\mathrm{ZnO}$. Having found the existing theories to be inadequate, he introduced the concept of the existence of the space-charge region at the interface, caused by the charge density gradient (or potential gradient) due to the non-passive nature of the different components present in the mixture, at the interface. Following Wagner, Crosbie $(1977,1978)$ studied the electrical conduction in $\mathrm{TiO}_{2}-\mathrm{SiO}_{2}$ mixtures and explained the data using the equation:

$$
\sigma \text { (dispersed solid) } / \sigma(\text { host })=\left\{1+0.83 g|Z| V_{v} \lambda / r_{1}^{2}\right\} .
$$

Here, $\sigma$ (dispersed solid) is the conductivity of the biphasic mixture, $\sigma$ (host) is the

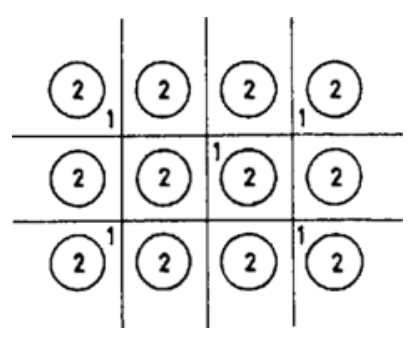

Figure 5. Rayleigh's model for two-phase systems. 
conductivity of the pure host matrix; $g$ is a structural factor $20 ; Z$ is the effective charge on the defects created; $V_{v}$ is the volume fraction of dispersoid in the mixture; $r_{1}$ is the radius of the dispersoid particle and $\lambda$ is the Debye length given by

$$
\lambda=\left(8 \pi e^{2} c(\infty) / \varepsilon k T\right)^{1 / 2},
$$

where $\varepsilon$ is the dielectric constant of the medium at temperature $T, k$ is the Boltzmann constant, $c(\infty)$ is the defect concentration in the bulk and $e$ is the fundamental charge.

Jow and Wagner (1979) have extended this approach to explain the enhancement of ionic conductivity in $\mathrm{CuCl}-\mathrm{Al}_{2} \mathrm{O}_{3}$. Akin to Wagner's approach, these authors also propose that ultrafine dispersoids (phase- $A$ ) form well defined space-charge regions in contact with the host electrolyte (phase- $M X$ ). Sphericity of the dispersoid particles assumed in the model (figure 6) gets support from the electron microscopy studies (Shahi and Wagner 1982a). An increase in defect concentration in this space-charge region enhances the ionic conductivities of the electrolyte. The total conductivity of the biphasic mixture including the contribution from the space-charge (sC) region $\sigma_{\mathrm{SC}}$, and neglecting the conductivity of insulting phase- $A$, is given by

$$
\sigma=\sigma_{0}+\sigma_{\mathrm{Sc}}
$$

$\sigma_{\mathrm{SC}}$, the space-charge region contribution, is calculated between distances $r_{1}$ and $r_{2}$ as shown in figure $6(\mathrm{c})$. In the spherical polar coordinates $\sigma_{\mathrm{SC}}$ will amount to:

$$
\sigma_{\mathrm{SC}}=\frac{\sum_{i} e \mu_{i}\left\{\int_{r_{1}}^{r_{2}} \int_{0}^{\pi / 2} \int_{0}^{2 \pi}\left[n_{i}(r)-n_{i}(\infty)\right] r^{2} \mathrm{~d} r \sin \theta \mathrm{d} \theta \mathrm{d} \phi\right\}}{\int_{r_{1}}^{r_{2}} \int_{0}^{\pi / 2} \int_{0}^{2 \pi} r^{2} \mathrm{~d} r \sin \theta \mathrm{d} \theta \mathrm{d} \phi} .
$$

(a)

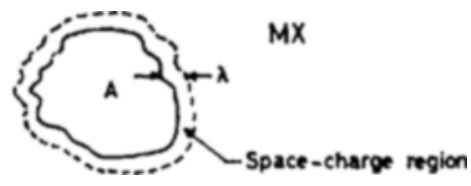

(b)

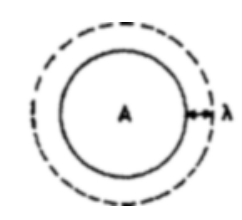

$\mathrm{MX}$

$M x$

(c)
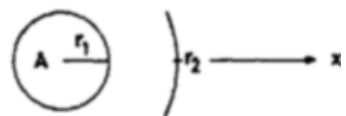

Figure 6. (a) Particle of $A$-phase embedded in $M X$-material and bearing a space-charge layer around it, (b) spherical approximation to the shape of $A$-phase particie, and (c) schematic cross-sectional view of a single $A$-phase particle of radius, $r_{1}$, in the matrix, $M X$. 
Substituting in (6) for $\sigma_{\mathrm{SC}}$ from (7) gives for the total conductivity

$$
\sigma=\sum_{i} n_{l}(\infty) e \mu_{i}+\sum_{i} e \mu_{i} \frac{\left\{\int_{r_{2}}^{r_{2}} \int_{0}^{\pi / 2} \int_{0}^{2 \pi}\left[n_{i}(r)-n_{i}(\infty)\right] r^{2} \mathrm{~d} r \sin \theta \mathrm{d} \theta \mathrm{d} \phi\right\}}{\int_{r_{1}}^{r_{2}} \int_{0}^{\pi / 2} \int_{0}^{2 \pi} r^{2} \mathrm{~d} r \sin \theta \mathrm{d} \theta \mathrm{d} \phi} .
$$

Here, $\mu_{i}$ is the mobility of the ith defect species; $n_{i}(r)$ and $n_{i}(\infty)$ are the defect concentrations at a point $r$ and in the bulk, respectively. The summation runs over all the different defect species. For $\lambda \ll r_{1}$, that is when the space-charge layer is much smaller than the size of the dipersoid particles the excess defect concentration in (8) is taken to be the arithmetic mean, $\left\langle\Delta n_{i}\right\rangle$, of the defect concentration at the surface and the bulk. Thus, $\sigma_{\mathrm{SC}}$ reduces to

$$
\sigma_{\mathrm{SC}}=\sum_{i} e \mu_{i}\left\langle\Delta n_{i}\right\rangle 4 \pi r_{1}^{2} \lambda /(4 \pi / 3)\left(r_{2}^{3}-r_{1}^{3}\right) .
$$

Assuming that $\left(r_{1} / r_{2}\right)^{3}=V_{v}$ the volume fraction of the dispersoid, (9) becomes

$$
\sigma_{\mathrm{SC}}=3 \sum_{i} e \mu_{i}\left\langle\Delta n_{i}\right\rangle\left(\lambda / r_{1}\right)\left(V_{\mathrm{v}} / 1-V_{v}\right)
$$

Equation (10) gives only a qualitative indication of the temperature dependence of dispersed solid-electrolytes. The enhancements observed in ionic conductivities of dispersed-solid electrolyte systems in relation to the pure host matrices are larger at low temperatures than those at high temperatures. This is envisaged by (10) since the Debye length, $\lambda$, decreases with increasing temperature. Equation (10) also explains the decrease in conductivity with increase in particle size of the dispersoid in conformity with the experimental findings (figure 3). This model is limited to small concentrations of the dispersoid and does not predict a maximum in conductivity of the dispersed solidelectrolyte at a particular concentration of the dispersoid as observed experimentally (figure 2). Besides, this model does not explain the mechanism leading to the enrichment of defect concentration in the space-charge regions of the various dispersed solid-electrolytes.

Recently, Maier (1984, 1985a, b) has proposed an approach for estimating the bulk conductivity of a dispersion of an insulator phase- $A$, in an ionically conducting matrix, phase-MX, employing the principle of parallel-switching (Maier 1985a). The physical situation adopted to estimate the total conductivity of the dispersed solid-electrolyte system is shown in figure 7. This model accounts for some of the inadequacies in the approach due to Jow and Wagner (1979). The model treats the space-charge region as a separate phase and considers each phase as a parallel resistor. The total conductivity, $\sigma$, of a dispersed solid-electrolyte given by

$$
\sigma=\beta_{1} \phi_{A} \sigma_{A}+\beta_{\infty} \phi_{\infty} \sigma_{\infty}+\beta_{\mathrm{SC}} \phi_{\mathrm{SC}} \sigma_{\mathrm{SC}},
$$

where, $\infty$ denotes the bulk $M X ; \phi_{i}$ is the volume fraction of phase $i ; \beta_{i}$ is a parameter describing the deviation from the ideal parallel-switching.

It would be appropriate to discuss the underlying defect chemistry of the dispersed solid-electrolytes to rationalize Maier's approach. Formation of Frenkel defects in a solid involves two steps (figure 8a). In the presence of the phase- $A$ which does not react 


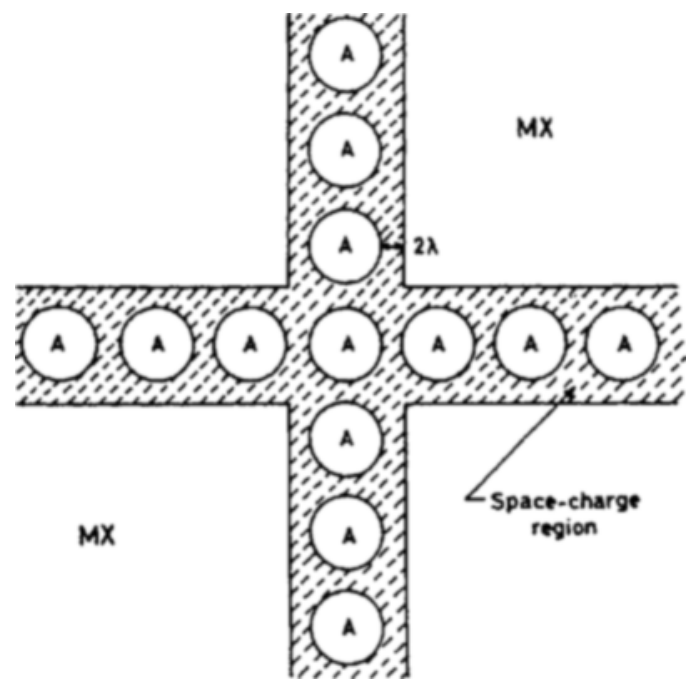

Figure 7. Coherent $A$-spheres forming crossing chains in the parallel-resistor model of Maier (1985a).

chemically with $M X$, the defect equilibrium may be affected. Attraction of metal ions $(M)$ from the phase- $M X$ by the chemical species present in phase $A$ leads to enrichment of surface vacancies through step 2 of figure $8 b$. The process of attraction of the $M$-ions from the host electrolyte $M X$ to phase- $A$ proceeds in the manner shown in figure $9 \mathrm{a}$. A repulsion of $M$-ions on the surface of $M X$ by the chemical species on phase- $A$ is equally feasible as shown in figure $9 \mathrm{~b}$. This would also lead to enrichment of surface vacancies as the forward reaction in step 2 of figure $8 \mathrm{a}$ will be more favoured. These attraction and repulsion processes are discrete and only one of them occurs for a given system under specific conditions. It was indicated earlier in our discussion that hydrated$\mathrm{Al}_{2} \mathrm{O}_{3}$ dispersoid leads to a larger enhancement in conductivities than the predried $\mathrm{Al}_{2} \mathrm{O}_{3}$ (figure 2). This could be due to attraction of $\mathrm{Ag}^{+}$ions by the hydroxyl groups present in hydrated $\mathrm{Al}_{2} \mathrm{O}_{3}$. In Schottky solids, however, a different situation exists. The

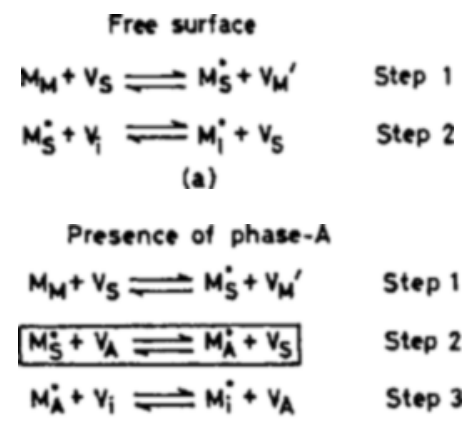

(b)

Figure 8. Defect formation in Frenkel solids: ( $\boldsymbol{a}$ ) on a free surface and (b) in presence of an insulator phase- $A$. 


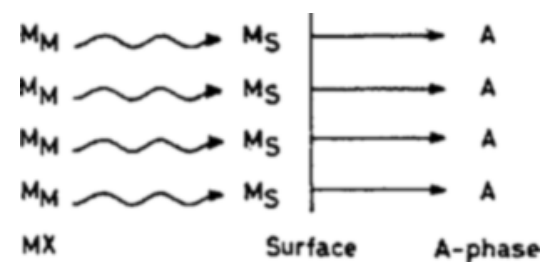

(a)

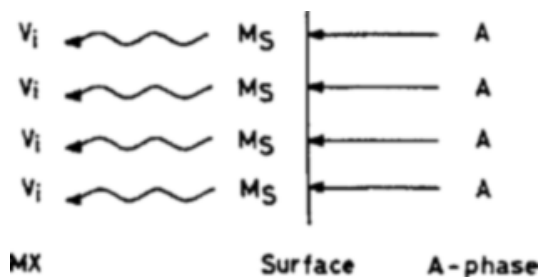

(b)

Figure 9. (a) Attraction mechanism by phase- $A$, and (b) Repulsion mechanism by $A$-phase.

defect equilibrium for formation of Schottky pairs is given in figure 10. In the presence of phase-A both cations and anions will compete for attraction and repulsion. Consequently, these two processes will be non-discrete in nature. It thus becomes difficult to predict categorically whether the conductivity of a solid with Schottky defects will be enhanced by a dispersed solid. On the basis of the above discussion, it becomes quite evident that the presence of the dispersoid phase- $A$ in a Frenkel solid would al ways lead to enrichment of surface vacancies in the space-charge region but not necessarily so in a Schottky solid.

The electrical conductivity component due to the space-charge region obtained by Maier by the exact integration of (8) in one-dimension cartesian co-ordinates using an exponential variation of the defect concentration is

$$
\sigma_{\mathrm{SC}}=e(2 \lambda) \mu_{v}\left(C_{v 0} C_{v v_{0}}\right)^{1 / 2} \text {. }
$$

Here, $\mu_{v}$ is the mobility of vacancies; $C_{v 0}$ and $C_{v \infty}$ are the concentrations of vacancies at the surface and the bulk, respectively; $2 \lambda$, the length factor is assumed to be the thickness of the space-charge layer. The dispersoid particles are assumed to be spherical and surrounded by a spherical space-charge region of thickness $2 \lambda$. The volume of the space-charge region is obtained by subtracting the volume of inner sphere from that of

$$
\begin{gathered}
M_{M}+v_{S} \rightleftharpoons v_{M}^{\prime}+{M_{S}^{\prime}}^{\prime} \\
x_{X}+v_{S} \rightleftharpoons x_{S}^{\prime}+v_{X}^{\prime} \\
M_{M}+x_{X}+2 v_{S} \rightleftharpoons v_{M}^{\prime}+v_{X}^{\dot{ }}+M_{S}^{\dot{1}}+x_{S}^{\prime}
\end{gathered}
$$

Figure 10. Formation of cation and anion vacancy pairs in Schottky solids. 
the outer sphere and is given by

$$
\sigma_{\mathrm{SC}}=3 \times\left(2 \lambda / r_{A}\right) \phi_{A}
$$

where $r_{A}$ is the radius of the dispersoid particles. The total conductivity from (11) is thus given by

$$
\sigma=\left(1-\phi_{A}\right) \sigma_{\infty}+3 e \beta_{S C} 2 \lambda\left(\phi_{A} / r_{A}\right) \mu_{v}\left(C_{v 0} C_{v \infty}\right)^{1 / 2}
$$

Here, the conductivity due to the insulating phase- $A$ has been neglected.

Equation (14) has been found to fit the experimental data of various dispersed solidelectrolytes fairly well, by adjusting the degree of non-ideal parallel-switching parameter, $\beta_{\text {sC }}$. Figure 11 shows the fit for $\mathrm{CaF}_{2}-\mathrm{CeO}_{2}$ and $\mathrm{CaF}_{2}-\mathrm{Al}_{2} \mathrm{O}_{3}$ systems (Vaidehi et al 1986). Similar quantitative fits have been obtained for $\mathrm{AgCl}-\mathrm{Al}_{2} \mathrm{O}_{3}$ and $\mathrm{AgCl}-\mathrm{SiO}_{2}$ (Maier 1985a) as well. The larger conductivity enhancements at low temperatures as compared to high temperatures are brought out correctly by (14). It is also obvious from (14) that as the particle size $\left(r_{A}\right)$ of the dispersoid increases, the conductivity decreases as observed experimentally. The attractive feature of Maier's model is that it highlights the mechanism responsible for enrichment of surface

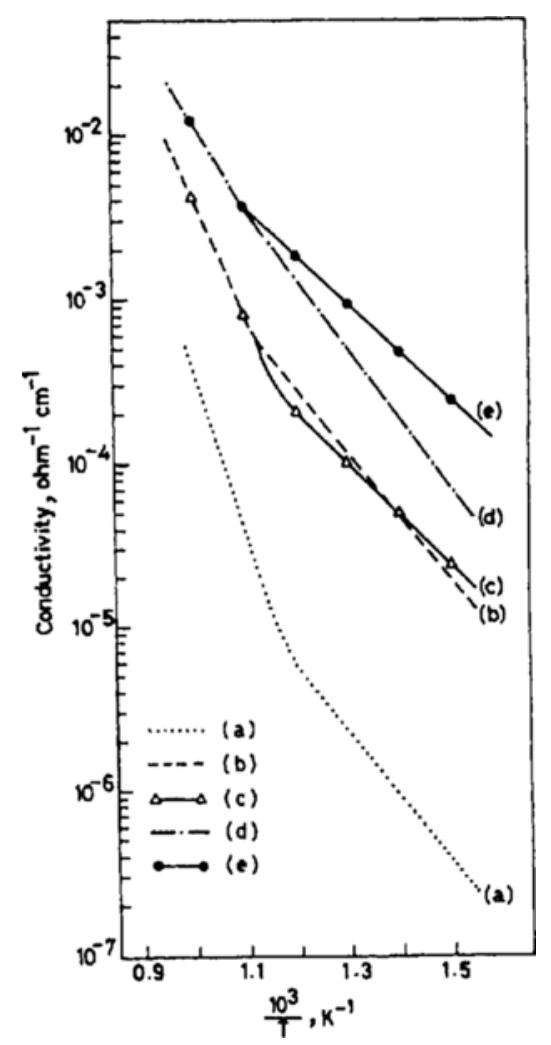

Figure 11. Yariation of conductivity with temperature for: (a) pure $\mathrm{CaF}_{2}$, (b) calculated values for $\mathrm{CaF}_{2}-2 \mathrm{~m} / \mathrm{o} \mathrm{Al} \mathrm{O}_{3}$, (c) experimental values for $\mathrm{CaF}_{2}-2 \mathrm{~m} / \mathrm{o} \mathrm{Al} \mathrm{O}_{3}$ (d) calculated values for $\mathrm{CaF}_{2}-2 \mathrm{~m} / \mathrm{o} \mathrm{CeO}$, and (e) observed values for $\mathrm{CaF}_{2}-2 \mathrm{~m} / \mathrm{o} \mathrm{CeO}$. 
vacancies in the space-charge region. Although this model is more quantitative than that due to Jow and Wagner, it has the same limitations.

Ionic conduction in dispersed solid-electrolytes has also been explained by Stoneham et al (1979). This model is an extension of Landauer's model (Landauer 1952) for the conductivity of a random mixture of two media in good electrical contact and with differing conductivities (figure 12). If the two media are labelled 1 and 2, one considers in turn the polarization of a sphere of each in an average medium. The average medium itself is taken to have consistently chosen conductivity appropriate to the aggregate of the two components. In dispersed solid electrolytes the situation is different. The model of Stoneham et al clearly denotes that the host electrolyte particles far from any particle of the insulating dispersoid should constitute one of the media and also that the model of the other component should recognize that the highly conducting boundary layer lies on a non-conducting core. The model also considers a structured sphere for calculating the polarization of the dispersoid particle including the boundary layer. It accounts for the concentration of the dispersoid in the host electrolyte. The spatial distribution of the conductivity, $\sigma$, near the dispersoid particle of radius, $R$, in a host matrix due to Stoneham et al (1979) is shown in figure 13a. According to Landauer's model, the conductivity of the system will not go to zero at any stage as there is no insulating phase present in biphasic metal mixtures. As has been indicated already, Landauer's approach is inadequate for application to dispersed solid-electrolyte systems due to a basic difference in the nature of the system itself. Taking the thickness of the space-charge region to be $t$, Stoneham et al found the conductivity of a dispersedsolid-electrolyte to vary as shown in figure $13 \mathrm{~b}$ near the insulating dispersoid particle. Stoneham et al also suggested that it would be appropriate to envisage a screening-layer model in these systems and in such a situation the conductivity will vary continuously as shown in figure $13 \mathrm{c}$, which is more realistic.

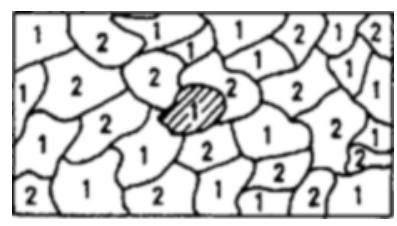

Figure 12. Landauer's polarization model.
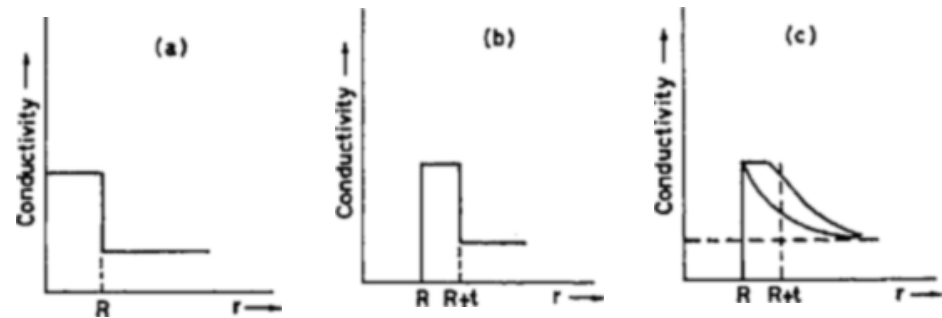

Figure 13. Spatial distribution of conductivity near an A-pbase insulator particle of radjus, $R$ : (a) Landauer model of bimetallic mixtures, (b) Discrete-shell model due to Stoneham et al (1979) for dispersed solid-electrolytes, and (c) screening-layer model. 
Although, the model due to Stoneham et al successfully predicts that at high concentrations of the dispersoid a saturation in conductivity of the solid electrolyte should be observed, it does not account for the influence of temperature on the conductivity behaviour of the system.

\section{Conclusions}

No single theory successfully explains all the characteristic features of the dispersedsolid-electrolyte systems. It seems imperative to explore a model which could account for all the features of these dispersed solid-electrolytes. Such a model might provide a tool for tailoring newer and probably better solid ionic conductors. Experimental studies for estimating the conductivity contribution from the space-charge region would be most desirable. Calorimetric measurements (Khandhar et al 1984) have shown that the space-charge region is associated with a large excess enthalpy in the AgI$\mathrm{AgBr}$ system. Measurement of the frequency dispersion of impedance in dispersed solid-electrolytes may be useful in estimating the conductivities of the space-charge region.

\section{Acknowledgements}

The authors are grateful to Professor C N R Rao for his interest and encouragement for writing this paper. Financial support from the Department of Science and Technology, Government of India, is gratefully acknowledged.

\section{References}

Bradley J N and Greene P D 1966 Trans. Faraday Soc. 622069

Bradley J N and Greene P D 1967 Trans. Faraday Soc. 63424

Chang M R W 1978 Master's thesis, Arizona State University

Crosbie G 1977 Ph.D. thesis, Northwestern University

Crosbie G 1978 J. Solid State Chem. 25367

Fujitsu S, Miyayama M, Koumoto K, Yanagida H and Kanazawa T 1985 J. Mater. Sci. 202103

Goodenough J B, Hong H Y P and Kafalas J A 1976 Mater. Res. Bull. 11203

Hong H Y P 1978 Mater. Res. Bull. 13117

Jander W 1929 Z. Angew. Chem. 42462

Jow T and Wagner Jr. J B 1979 J. Electrochem. Soc. 1261963

Khandhar A and Wagner Jr. J B 1983 Extended Abstract 833 Eloctrochemical Society Meeting, San Francisco (North Hampshire: Electrochemical Society)

Khandhar A, Tare V B, Navrotsky A and Wagner Jr. J B 1984 J. Electrochem. Soc. 1312683

Landauer R 1952 J. Appl. Phys. 23779

Liang C C 1973 J. Electrochem. Soc. 1201289

Lichtenecker K 1924 Phys. Z. 25225

Maier J 1984 Phys. Status Solidi B123 K89

Maier J 1985a J. Phys. Chem. Solids 46309

Maier J 1985b Mater. Res. Bull. 20383

Maxwell J C 1965 Electricity and magnetism (London: Heywood)

Owens B B and Argue G R 1967 Science 157308

Pack S 1979 Abstract 133 Electrochemical Society Meeting, Boston (North Hampshire: Electrochemical Society) 
Pack S, Owens B and Wagner Jr. J B 1980 J. Electrochem. Soc. 1272177

Rayleigh 1892 Philos. Mag. 34481

Reuter B and Hardel K 1961 Naturwissenschaften 48161

Reuter B and Hardel K 1965 Z. Anorg. Allg. Chem. 340158

Rickert H 1982 Electrochemistry of solids: an introduction (Berlin, Heidelberg: Springer Verlag)

Shahi K and Wagner Jr. J B 1981 a Solid State Ionics 3/4 295

Shahi K and Wagner Jr. J B 1981 b J. Electrochem. Soc. 1286

Shahi K and Wagner Jr. J B 1982a J. Solid State Chem. 42107

Shahi K and Wagner Jr. J B 1982b J. Phys. Chem. Solids 43713

Stoneham A M, Wade E and Kilner J A 1979 Mater. Res, Bull 14661

Strock L W 1934 Z. Phys. Chem. B25 441

Strock L W 1935 Z. Phys. Chem. B31 132

Vaidehi N, Akila R, Shukla A K and Jacob K T 1986 Mater. Res. Bull. (in press)

Wagner C 1972 J. Phys. Chem. Solids 331051

Wagner J B Jr 1980 Mater. Res. Bull. 151961

Weber N and Kummer J T 1967 Proc. Ann. Power Sources Conf. 2137 